\title{
Modelling second malignancy risks from low dose rate and high dose rate brachytherapy as monotherapy for localised prostate cancer
}

DOI:

10.1016/j.radonc.2016.05.026

\section{Document Version}

Accepted author manuscript

Link to publication record in Manchester Research Explorer

Citation for published version (APA):

UroGEC/BRAPHYQS group of the GEC ESTRO (2016). Modelling second malignancy risks from low dose rate and high dose rate brachytherapy as monotherapy for localised prostate cancer. Radiotherapy and oncology : journal of the European Society for Therapeutic Radiology and Oncology, 120(2), 293-9.

https://doi.org/10.1016/j.radonc.2016.05.026

\section{Published in:}

Radiotherapy and oncology : journal of the European Society for Therapeutic Radiology and Oncology

\section{Citing this paper}

Please note that where the full-text provided on Manchester Research Explorer is the Author Accepted Manuscript or Proof version this may differ from the final Published version. If citing, it is advised that you check and use the publisher's definitive version.

\section{General rights}

Copyright and moral rights for the publications made accessible in the Research Explorer are retained by the authors and/or other copyright owners and it is a condition of accessing publications that users recognise and abide by the legal requirements associated with these rights.

\section{Takedown policy}

If you believe that this document breaches copyright please refer to the University of Manchester's Takedown Procedures [http://man.ac.uk/04Y6Bo] or contact uml.scholarlycommunications@manchester.ac.uk providing relevant details, so we can investigate your claim.

\section{OPEN ACCESS}




\section{UNIVERSITY OF LEEDS}

This is a repository copy of Modelling second malignancy risks from low dose rate and high dose rate brachytherapy as monotherapy for localised prostate cancer.

White Rose Research Online URL for this paper:

http://eprints.whiterose.ac.uk/101262/

Version: Accepted Version

\section{Article:}

Murray, L orcid.org/0000-0003-0658-6455, Mason, J, Henry, AM

orcid.org/0000-0002-5379-6618 et al. (4 more authors) (2016) Modelling second malignancy risks from low dose rate and high dose rate brachytherapy as monotherapy for localised prostate cancer. Radiotherapy and Oncology, 120 (2). pp. 293-299. ISSN 0167-8140

https://doi.org/10.1016/j.radonc.2016.05.026

(C) 2016, Published by Elsevier Ireland Ltd. Licensed under the Creative Commons Attribution-NonCommercial-NoDerivatives 4.0 International http://creativecommons.org/licenses/by-nc-nd/4.0/

\section{Reuse}

Unless indicated otherwise, fulltext items are protected by copyright with all rights reserved. The copyright exception in section 29 of the Copyright, Designs and Patents Act 1988 allows the making of a single copy solely for the purpose of non-commercial research or private study within the limits of fair dealing. The publisher or other rights-holder may allow further reproduction and re-use of this version - refer to the White Rose Research Online record for this item. Where records identify the publisher as the copyright holder, users can verify any specific terms of use on the publisher's website.

\section{Takedown}

If you consider content in White Rose Research Online to be in breach of UK law, please notify us by emailing eprints@whiterose.ac.uk including the URL of the record and the reason for the withdrawal request. 
Modelling second malignancy risks from Low Dose Rate and High Dose Rate brachytherapy as monotherapy for localized prostate cancer.

Louise Murray, ${ }^{a, b}$, Joshua Mason ${ }^{a}$, Ann M Henry ${ }^{a, b}$, Peter Hoskinc, Frank-Andre Siebert ${ }^{d}$, Jack Venselaare, Peter Bownes ${ }^{\mathrm{a}}$; on behalf of the UroGEC/BRAPHYQS group of the GEC ESTRO.

aDepartments of Radiotherapy and Medical Physics, St James's Institute of Oncology and bUniversity of Leeds, UK; 'Mount Vernon Cancer Centre, Northwood, UK; ${ }^{\text {dKlinik fur }}$

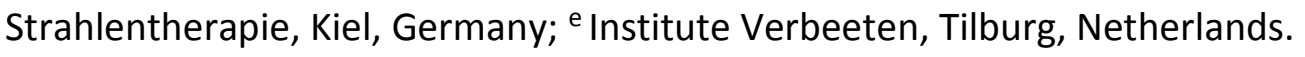

Address for correspondence:

Dr Ann M Henry

St James's Institute of Oncology

Beckett St, Leeds, LS9 7TF, UK

Email:a.henry@leeds.ac.uk

Short title: Brachytherapy second cancer risks 


\section{Abstract \\ Background and Purpose}

To estimate the risks of radiation-induced rectal and bladder cancers following low dose rate (LDR) and high dose rate (HDR) brachytherapy as monotherapy for localized prostate cancer and compare to external beam radiotherapy techniques.

\section{Materials and Methods}

LDR and HDR brachytherapy monotherapy plans were generated for three prostate CT datasets. Second cancer risks were assessed using Schneider's concept of organ equivalent dose. LDR risks were assessed according to a mechanistic model and a bell-shaped model. HDR risks were assessed according to a bell-shaped model. Relative risks and excess absolute risks were estimated and compared to external beam techniques.

\section{Results}

Excess absolute risks of second rectal or bladder cancer were low for both LDR (irrespective of the model used for calculation) and HDR techniques. Average excess absolute risks of rectal cancer for LDR brachytherapy according to the mechanistic model were 0.71 per 10,000 person-years (PY) and 0.84 per 10,000 PY respectively, and according to the bellshaped model, were 0.47 and 0.78 per 10,000 PY respectively. For HDR, the average excess absolute risks for second rectal and bladder cancers were 0.74 and 1.62 per 10,000 PY respectively. The absolute differences between techniques were very low and clinically irrelevant. Compared to external beam prostate radiotherapy techniques, LDR and HDR brachytherapy resulted in the lowest risks of second rectal and bladder cancer.

\section{Conclusions}

This study shows both LDR and HDR brachytherapy monotherapy result in low estimated risks of radiation-induced rectal and bladder cancer. LDR resulted in lower bladder cancer risks than HDR, and lower or similar risks of rectal cancer. In absolute terms these differences between techniques were very small. Compared to external beam techniques, second rectal and bladder cancer risks were lowest for brachytherapy.

Keywords: High Dose Rate Brachytherapy, Localised prostate cancer, Low Dose Rate Brachytherapy, Second Cancer Risks 


\section{Introduction}

The diagnosis of a radiation-induced second primary cancer is a recognised late complication following radiotherapy treatment. Patients who survive for many years following radiotherapy are thought to be at greatest risk, and so for younger prostate cancer patients, the risk of second malignancy is a relevant issue, particularly when a range of treatment modalities are available. Clinical studies have attempted to address the second cancer risks associated with the traditional external beam techniques used in prostate cancer 1. For more modern radiation techniques such as brachytherapy and IMRT, where insufficient clinical follow-up and lower patient numbers mean that valid conclusions cannot yet be drawn, planning studies and theoretical modelling have attempted to provide answers instead 2. There is, however, very little data, clinical or theoretical, which address the risks of second malignancy following brachytherapy monotherapy. Brachytherapy monotherapy is a possible treatment option for many patients with early prostate cancer, and so assessments of the risk of radiation-induced second malignancy would help inform the decision making process when patients are considering which treatment option to undertake.

This paper aims to investigate second malignancy risks associated with both low dose rate (LDR) and high dose rate (HDR) brachytherapy monotherapy using Schneider's concept of organ equivalent dose 3. Risks are compared with previously published work regarding second malignancy risk from external beam techniques [4]. This study was undertaken in the framework of the GEC ESTRO UroGEC/BRAPHYQS group.

\section{Methods}

\section{Contouring}

Three prostate CT datasets for patients originally treated using external beam radiotherapy were selected at random. All patients had enemas prior to scanning to ensure that rectums were empty. The prostate, bladder, and rectum were contoured. A $5 \mathrm{~mm}$ shrink margin was created within the bladder and the subtraction of this structure from the bladder structure 
was used to represent the bladder wall for assessment of radiation-induced bladder cancer. The risk of radiation-induced rectal cancer was calculated form the whole (empty) rectal structure.

\section{Planning}

For each of the three datasets, an LDR and HDR plan was created. The CT planning scans were rotated so that the position of the prostate approximated that used in trans-rectal ultrasound based treatment planning (flat posterior prostate capsule). LDR plans used stranded AgX100 (Theragenics Corporation, Buford, GA) iodine-125 seeds with air-kerma strength $0.453 \mathrm{U}$ and prescribed dose of $145 \mathrm{~Gy}$ to the $100 \%$ isodose, as per ESTRO recommendations 5]. HDR plans used the iridium-192 Flexisource (Elekta AB, Stockholm, Sweden) with $2 \mathrm{~mm}$ dwell spacing. HDR treatments are prescribed to the planned prostate D90 with 19Gy as the $100 \%$ isodose and delivered as a single fraction 6. LDR plans were created in Variseed ${ }^{\mathrm{TM}}$ v8.0 (Varian Medical Systems, Inc., Palo Alto, CA) and HDR plans were created in Oncentra Prostate ${ }^{\mathrm{TM}}$ v4.1.3 (Elekta $A B$ ). Treatment planning algorithms using the TG-43 formalism were used 77. The prostate volumes used in this study were 22.7, 32.8 and $34.5 \mathrm{~cm}^{3}$; although in practice a wider range of prostate volumes would be considered appropriate for HDR brachytherapy. Table 1 summarises the planning objectives and mean achieved plan DVH parameters for each plan type.

Differential dose volume-histograms (DVHs) for the rectum, and bladder wall were exported for second malignancy risk calculation using a bin width of $0.1 \mathrm{~Gy}$.

\section{Second malignancy risk estimation}

Schneider's concept of Organ Equivalent Dose (OED) was used to compare and estimate the risk of radiation-induced second malignancy from brachytherapy monotherapy 3.8. The OED concept and calculation process has been described in detail elsewhere 38 . In brief, two different radiotherapy plans, which result in the same risk of second malignancy, have the same OED. The OED of one plan relative to another therefore gives the relative risk of second malignancy for those two techniques. The OED concept can be used to calculate 
second malignancy risk using Schneider's mechanistic model which incorporates the impact of fractionation as well as a tissue specific repair/ repopulation factor, $R$ 9. The OED concept can also be used to demonstrate two extreme possibilities 8.:

i) the situation of no repair/ repopulation- this is a bell-shaped model of radiation-induced malignancy whereby the risk of second cancer initially increases with increasing doses but, after a certain point, cells become sterilised and no longer have the potential for malignant transformation, and so the risk of second cancer then begins to reduce (here the effect of fractionation is removed, i.e. $R=0$ )

ii) the situation of full repair- this is a plateau model of radiation-induced malignancy whereby risk of second cancer initially increases with increasing dose, and then at some point levels off as all damaged cells are repaired, resulting in a constant second cancer risk above a certain threshold (in this situation $R=1$ ).

A bell-shaped model for OED calculation was used for the evaluation of the LDR and HDR brachytherapy plans assuming no repair or repopulation takes place, thereby removing the effect of fractionation or protraction 8. To include the effects of long treatment duration, the alternative of the mechanistic model was used as well for the LDR plan.

For the bell-shaped model the OED is calculated according to Equation 1 [8:

1)

$$
\mathrm{OED}=\frac{1}{\mathrm{~V}_{\mathrm{T}}} \sum_{\mathrm{i}} \mathrm{V}_{\mathrm{D}_{\mathrm{i}}} \mathrm{RED}_{\mathrm{D}_{\mathrm{i}}}
$$

where $V_{T}$ is the total volume of a structure, $V_{D}$ is the volume of dose bin $i$ receiving dose $D$, and the RED is the risk equivalent dose for that dose bin. For each dose bin using a bellshaped model, RED is calculated according to Equations 2 and 38 : 
2)

$$
R E D_{D}=\mathrm{D} \exp (-\alpha \mathrm{D}), \quad \text { and }
$$

3)

$$
\alpha^{\prime}=\alpha+\beta d=\alpha+\beta\left(D / D_{T}\right) d_{T}
$$

where $D$ is the dose received by the DVH bin, $D_{T}$ is the dose prescribed to the target and $d_{T}$ is the prescribed dose per fraction to the target. In the case of brachytherapy monotherapy, $D_{T}$ and $d_{T}$ were the same for each bin of the DVH since the number of fractions was one. Values for $\alpha$ were derived by Schneider et al 8, based on patients irradiated for Hodgkin's disease and atomic bomb survivors, and those used in this study are shown in Table 2. In all cases, $\beta$ was such that $\alpha / \beta=3 \mathrm{~Gy}$.

Although LDR is delivered as a single fraction, since the dose rate is low, it is accepted that a degree of normal tissue repair occurs during LDR dose delivery 10 given the low dose rate of delivery (i.e. for I-125 LDR as used here, 90\% of the dose is delivered over 204 days). Alternatively, to incorporate potential normal tissue repair during LDR delivery into the risk estimation, OED was also calculated using Schneider's mechanistic model 8 89. Here using Equation 4:

4)

$$
R E D_{D}=\frac{\mathrm{e}^{-\alpha^{\prime} \mathrm{D}}}{\alpha^{\prime} \mathrm{R}}\left(1-2 \mathrm{R}+\mathrm{R}^{2} \mathrm{e}^{\alpha^{\prime} \mathrm{D}}-(1-\mathrm{R})^{2} \mathrm{e}^{-\frac{\alpha^{\prime} \mathrm{R}}{1-\mathrm{R}} \mathrm{D}}\right)
$$

where $\alpha^{\prime}$ is calculated as above, and $R$ is a tissue specific repair/ repopulation parameter (Table 2).

The excess absolute risk (EAR) of developing a radiation-induced cancer can be calculated using the RED according to Equation 5 :

5) $\quad$ EARorg $=\frac{1}{\mathrm{~V}_{\mathrm{T}}} \sum_{\mathrm{i}} \mathrm{V}_{\mathrm{D}_{\mathrm{i}}} \bullet \mathrm{RED}_{\mathrm{D}_{\mathrm{i}}} \bullet \beta_{\text {EAR }} \bullet \mu($ agex, agea $)$ 
where $V_{T}$ is the total volume of a structure, $R E D_{D i}$ and $V_{D}$ are as above, $\beta_{E A R}$ is the initial slope for the dose-risk curve for radiation-induced second cancer and $\mu$ takes into account the age of the population examined, based on agex, the patient age at the time of irradiation and agea, the attained age of the patient (years). All EAR calculations in this study were calculated for patients irradiated at age 60 years (agex) and attaining age 80 years (agea) as representative of the localised prostate cancer population at risk. The factor, $\mu$, was calculated according to Equation 6:

6)

$$
\mu(\text { agex }, \text { agea })=\exp \left(\gamma_{e}(\text { agex }-30)+\gamma_{a} x \ln (\text { agea } / 70)\right.
$$

where $\gamma_{e}$ and $\gamma_{a}$ are age modifying factors ( $\beta_{E A R}$ was originally calculated for persons exposed at age 30 years and attaining age 70 years). The parameters used for EAR calculations are shown in Table 2.

The calculated OEDs for LDR and HDR brachytherapy were compared with those calculated in a previous study for 3D-conformal radiotherapy (3D-conformal) delivered as 78Gy in 39 fractions using the same three prostate datasets 4 . The relative risk of second cancer from brachytherapy was estimated compared to this 3D-CRT treatment schedule. OEDs for brachytherapy were also compared to those previously calculated for other additional external beam techniques using the mechanistic model: IMRT (78Gy in 39 fractions), volumetric modulated arc therapy (VMAT; 78Gy in 39 fractions using flattened and flattening filter free (FFF) beams), and stereotactic ablative radiotherapy (SABR; 42.7Gy in 7 fractions delivered using flattened and FFF beams) 4 .

According to the models employed here, for single fraction brachytherapy, the risks of radiation-induced second rectal and bladder cancers peak around 9Gy and 2Gy respectively, before reducing steeply when using the bell-shaped model, and more gradually, when using the mechanistic model (as a degree of repair is permitted here). The shape of the doseresponse relationship is illustrated graphically in Supplementary Material. 


\section{Results}

According to the mechanistic model, LDR plans resulted in similar risks of second rectal cancer relative to $H D R$ plans (average relative risk $L D R_{\text {mech }}: H D R=0.97$, range: 0.90-1.03). According to the bell-shaped model, the risk of second rectal cancer was lower with LDR than HDR brachytherapy with an average relative risk of 0.64 (range: 0.53-0.77). Similarly, according to both mechanistic and bell-shaped models, the risk of second bladder cancer was about 50\% lower with LDR than HDR monotherapy (average relative risk $\mathrm{LDR}_{\text {mech }}: \mathrm{HDR}=0.53$ (range: 0.22-0.72), average relative risk $\mathrm{LDR}_{\text {bell }}: \mathrm{HDR}=0.50$ (range: 0.150.70). Regardless of whether mechanistic or bell-shaped models were used, the relative risk reduction with LDR monotherapy compared to HDR monotherapy was greater for second bladder cancers than rectal cancers (Fig. 1, where individual patient data is shown). In absolute terms, expressed in terms of the number of person-years, the risks of second rectal or bladder cancer were low for both LDR (irrespective of the model used for calculation) and HDR techniques (Fig. 2). For LDR, according to the mechanistic model, the average EARs for second rectal and bladder cancers were 0.71 per 10,000 person-years (PY; range: 0.68-0.78) and 0.84 per 10,000 PY (range: 0.37-1.12) respectively, and according to the bell-shaped model, were 0.47 and 0.78 per 10,000 PY (ranges: $0.40-0.51$ and $0.26-1.08$ ) respectively. For HDR, the average EARs for second rectal and bladder cancers were slightly higher at 0.74 and 1.62 per 10,000 PY (ranges: $0.66-0.80$ and 1.42-1.72) respectively. As would be expected, including a degree of repair and repopulation in the LDR calculations resulted in increased OEDs and higher EARs, although in absolute terms these differences were small (differences between average EARs using mechanistic and bell-shaped models for second rectal and bladder cancers were 0.24 and 0.05 per 10,000 PY respectively, and, based on individual patient data, largest differences between EARs using mechanistic and bell-shaped models for second rectal and bladders cancers were 0.28 per 10,000 PY and 0.11 per 10,000 PY respectively). Similarly, the absolute differences in EARs between HDR and LDR techniques were also low (at most 0.27 per 10,000 PY for second rectal cancer and 0.83 per 10,000 PY for second bladder cancer based on averaged EARs, and, based on individual 
patient data, at most 0.35 per 10,000 PY and 1.44 per 10,000 PY for second rectal and bladder cancers respectively).

Based on previously published work using the same three prostate datasets [4], OEDs for the brachytherapy techniques were compared to those calculated for 3D-CRT 78Gy in 39 fractions (Fig. 3, average and range for all three datasets shown for all techniques). Out of all the techniques examined, LDR and HDR brachytherapy resulted in the lowest risks of second rectal and bladder cancers relative to 3D-CRT. The excess absolute risks of second rectal and bladder cancers are illustrated for external beam techniques together with LDR and HDR techniques (Fig. 4, average and range for all three datasets shown for all techniques). The absolute risks of second rectal and bladder cancer were low for all external beam and brachytherapy techniques (highest average EARs for second rectal and bladder cancers: 2.7 per 10,000 PY (3D-CRT) and 2.4 per 10,000 PY (IMRT) respectively) but lowest for brachytherapy. Absolute differences between techniques were also low, at most 2.2 per 10,000 PY for rectal cancer (the difference between average EARs using 3D-CRT and LDR $\mathrm{B}_{\text {bell }}$ brachytherapy), and 1.6 per 10,000 PY for bladder cancer (the difference between average EARs using IMRT and LDR bell brachytherapy).

\section{Discussion}

Brachytherapy is one of several treatment options available to patients with localised prostate cancer. Patients may be treated with LDR or HDR brachytherapy. Clinical evidence

suggests that both techniques result in high rates of PSA control 11-15. As well as efficacy, the toxicity profiles of different techniques must also be considered, including the risk of second malignancy. Here we demonstrate that compared to external beam techniques, both LDR and HDR brachytherapy result in lower relative risks of second malignancy and very low absolute risks of second malignancy, although the absolute risks of second rectal and bladder cancer were low for all the techniques examined. LDR brachytherapy resulted in lower bladder cancer risks and lower or similar risks of rectal cancer relative to HDR brachytherapy according to the models used, but, in absolute terms, these differences were low. One might deduce that because of the very high (potentially cell sterilising) doses that are delivered to small volumes of normal tissues and because of the very rapid dose fall off 
that occurs with brachytherapy, second malignancy risks would be lower compared to external beam techniques, and the findings of this study support this.

Clinical studies have evaluated second malignancy in patients treated with brachytherapy (predominantly LDR) in comparison to the general population or compared to nonirradiated prostate cancer patients. Few comparisons have been made between patients irradiated with brachytherapy compared to patients irradiated with other techniques. Compared to the general (i.e. non-prostate cancer) population, one SEER registry study 16 and three single institution studies 17-19. found that the risk of rectal cancer following brachytherapy monotherapy or combination brachytherapy and external beam radiotherapy (BT-EBRT) was no higher than that in the general population. The risk of bladder cancer after brachytherapy monotherapy was also found to be no different to that in the general population in one SEER registry study though there was an increased risk in patients who received combination BT-EBRT16. Two single institution studies, one examining brachytherapy monotherapy and one examining combination BT-EBRT and brachytherapy monotherapy found no significant difference in the risk of bladder cancer compared to the general population 18 19. In contrast, two other single institution studies, one examining a mixed population of brachytherapy monotherapy and BT-EBRT patients, and one examining only brachytherapy monotherapy patients, found these patients to be at increased risk of bladder cancer compared to the general population $17 \mid 20$.

Compared to non-irradiated prostate cancer patients, three SEER analyses 16 21||22. two single institution studies 1723 . 23 found no increase in the risk of rectal cancer in brachytherapy monotherapy patients. For patients treated with combination BT-EBRT, two of three SEER analysis found no difference in the risk of rectal cancer compared to nonirradiated patients 21 22, while the largest SEER analysis, observed an increase in the risk of rectal cancer but only after 10 years of follow-up 16. Two single institution studies, one examining EBRT-BT patients specifically 23, and one examining a mixed population of brachytherapy monotherapy and combination BT-EBRT patients 24 observed no difference in the risk of rectal cancer compared to non-irradiated prostate cancer patients. In terms of bladder cancer, two SEER analyses $21 \mid 22$ and three single institution studies $17|23| 24$ examining both brachytherapy monotherapy and combination BT-EBRT patients found no 
difference in the risk of bladder cancer in comparison to non-irradiated patients. In contrast, a third and larger SEER analysis observed an increase in the risk of bladder cancer in both monotherapy and combination BT-EBRT patients compared to non-irradiated patients 16 .

More relevant for this study, are the three clinical studies (one SEER analysis 22. and two single institution studies 24 25,) that have compared second cancers in brachytherapy patients with prostate patients treated with external beam radiotherapy. The one SEER analysis observed no difference in the risk of second rectal and bladder cancer in patients treated with brachytherapy monotherapy or combination BT-EBRT compared to patients irradiated using external beam radiotherapy alone22. Similarly, neither of the single institution studies has observed any difference in the risk of rectal or bladder cancer, between patients irradiated using external beam radiotherapy and brachytherapy monotherapy patients and a mixed population of brachytherapy monotherapy and combination BT-EBRT patients 24 25. This is partly in contrast to this current work, which has suggested the risk of second rectal and bladder cancers may be lower than other external beam techniques. In all of the above clinical studies, however, the follow-up in brachytherapy patients is generally shorter than in studies examining second cancer risks in patients treated with external beam techniques. In addition, patient numbers in brachytherapy cohorts are generally lower than those examined in studies of second cancers in external beam patients. As such, an accurate picture regarding the second cancer risks following brachytherapy in comparison to the general population, non-irradiated prostate cancer patients and patients irradiated with external beam techniques cannot yet be formed. Furthermore, where combination BT-EBRT is examined, both the external beam and brachytherapy components will contribute to second cancer risk and the proportion of risk attributable to brachytherapy cannot be determined, adding to the difficulties in drawing a definitive conclusion about second cancer risk from brachytherapy.

In the absence of adequate clinical evidence, planning studies can be used to give an estimation of second cancer risk. Few studies, however, have examined the risks of second cancer from prostate brachytherapy. In fact only one study was identified which estimated 
second cancer risks from brachytherapy 26. Both LDR and HDR brachytherapy monotherapy were examined in addition to combination external beam radiotherapy with an HDR brachytherapy boost. This study estimated risks using the Competition model, a model which predicts maximal cancer inductions at doses of around 4Gy 27 . To allow comparisons between these different techniques, doses were normalised to the same biological end-point by calculating the biologically effective doses for the DVH bins by multiplying the physical dose by the relative effectiveness of each technique 26. It was found that the risk of rectal cancer was low with all techniques but lowest for brachytherapy monotherapy. Here the average lifetime risk of rectal cancer was $2.0 \times 10^{-4} \%$ for LDR monotherapy, $1.0 \times 10^{-4} \%$ for HDR monotherapy and $0.06 \%$ for combination external beam and HDR treatment 26. On average, therefore, HDR monotherapy resulted in lower rectal cancer risks than LDR monotherapy, although in absolute terms the difference was minimal and clinically insignificant. Despite the differences in modelling technique and the calculation of a lifetime risk rather than a risk per 10,000 PY, as used in this current piece of work, it can be concluded that both studies demonstrate that the estimated risks of radiation-induced rectal cancer are low and that the differences in second cancer risks between LDR and HDR brachytherapy are clinically insignificant.

There is much debate as to the most appropriate means of estimating second malignancy risks and a variety of models exist, none of which have been shown to be a perfect fit to the clinical data 28. In addition, the error associated with such models can be very large with EAR estimates resulting in errors of up to $100 \%$ (Equation 5), although relative risk calculations using OED are associated with lower errors at 5-10\% (Equation 1) 29 30. The complexity of the situation increases further when trying to use models that were designed for the analysis of external beam techniques, and which likely did not consider the impact of treatment with different dose rates or even the impact of high dose per fraction treatments. This may at least partly explain the paucity of planning studies that try to estimate second cancer risks form prostate brachytherapy. We examined single fraction HDR monotherapy. The dose rate here is similar to external beam treatments, upon which the OED concept was originally based. We used the bell-shaped model for these calculations, and assumed the effect of fractionation and repair/ repopulation is so small it can be neglected. For LDR brachytherapy monotherapy the situation is more difficult. Although treatment is delivered 
in a single fraction, supporting the use of a bell-shaped model, the lower dose rate delivery is thought to permit a degree of normal tissue repair[10, suggesting that the bell-shaped curve may underestimate the risk of second cancer and that a mechanistic model which includes an element of repair/ repopulation may be more appropriate. A degree of repair and repopulation will result in a proportion of normal cells becoming at risk of malignant transformation (instead of being completely sterilised), thus resulting in an increase in OED and second malignancy risk. In order to demonstrate both possibilities we opted to calculate risk for LDR monotherapy using both the bell-shaped and mechanistic models. We did not adopt a plateau model, which assumes full repair/ repopulation between fractions, as there would be no break in radiation dose delivery with LDR to permit full repair/ repopulation. According to the mechanistic model, the risk of rectal cancer was broadly similar between HDR and LDR treatments and the risk of bladder cancer lower with LDR. According to the bell-shaped model the risks of rectal and bladder cancers were lower with LDR compared to HDR monotherapy. Even if the relative differences between HDR and LDR brachytherapy were as large as the calculation according to the bell-shaped model suggests, in absolute terms the differences between LDR and HDR monotherapy treatments are small and clinically irrelevant. The authors accept that using the mechanistic model for a single fraction LDR treatment is not using the model in the manner in which it was originally designed, and so there are greater uncertainties associated with the risks estimated for LDR treatments than the other evaluated treatments. The use of the mechanistic model, however, does allow a degree of repair and repopulation to be included in the calculation, which intuitively seems appropriate given the low dose rate nature of treatment delivery.

This study has limitations. As mentioned above, there is uncertainty in all models of second cancer risk estimation, and this uncertainty is increased when applying these models to the setting of brachytherapy, particularly LDR brachytherapy. The optimal way to account for LDR irradiation (e.g. biologically effective dose transformation, OED calculation using a mechanistic model) in this setting is unknown. In addition, we did not look at pulsed dose rate Brachytherapy (PDR) brachytherapy as this is infrequently used for prostate cancer and is more complex given its fractionation (incorporation of repair effects). 
Only three prostate datasets were examined in this study, potentially limiting the generalisability of our findings. For prostate cancer, the positions of the rectum and bladder in relation of the prostate are relatively constant in comparison to other tumours such as lung cancers where the proximity to organs at risk can be very variable. As such, interpatient variation in second rectal and bladder cancer risks may be less than the inter-patient variation in the risk of other second cancers in patients with other, more anatomically variable, primary sites, although this will be influenced by the models used and radiotherapy delivery technique. Combination BT-EBRT treatments were not included as this would introduce further uncertainties into the risk estimations.

Other limitations of this work include that the brachytherapy DVHs used to estimate second malignancy risk would not be fully representative of the actual doses received by the normal tissues. For HDR treatments, the imaging modality used for plan calculation may be different to what we used (can be CT or US) and this could result in some small differences in the DVHs produced. Furthermore, in the case of LDR brachytherapy, the DVH is based on a preplan, and so is not the same as the delivered plan, generally assessed on CT +/- MRI, nor are the effects of seed migration or oedema incorporated into the DVH. For HDR treatments, dose may be delivered with the probe in place, which results in part of the rectum receiving lower doses than the doses represented by the DVHs used for risk calculations here (where the probe was not included during plan calculation). The use of the TG-43 formalisation for dose calculation in brachytherapy treatments has limitations as no account for tissue composition and inter-source absorption is made. Future planning systems may provide more accurate dosimetry with the use of Monte Carlo based methods 31 32. We anticipate, however that the impact of these inaccuracies, in terms of the calculation of the risk of second malignancy, would be small, and indeed the uncertainties introduced as a result of these inaccuracies are much smaller than the uncertainties associated with the second malignancy risk estimation process itself. 


\section{Conclusions}

Despite the limitations and uncertainties in the estimation, the modelled risk of second rectal and bladder cancer from brachytherapy monotherapy appears low. The relative risk of radiation-induced bladder cancer was lower from LDR brachytherapy compared to HDR brachytherapy. Depending on the model used, the relative risk of rectal cancer was similar or lower with LDR brachytherapy compared to HDR. In absolute terms, however, the differences were very small. Brachytherapy second rectal and bladder cancer risks are lower than the risks associated with external beam treatments. The clinical evidence regarding second cancer risk from brachytherapy is encouraging but immature, and so this study provides reassurance regarding the long-term safety of brachytherapy with regard to second malignancy risk.

Conflict of Interest Statement: None declared 


\section{References}

1. Murray L, Henry A, Hoskin P, Siebert FA, Venselaar J. Second primary cancers after radiation for prostate cancer: a systematic review of the clinical data and impact of treatment technique. Radiotherapy and oncology : journal of the European Society for Therapeutic Radiology and Oncology. 2014;110(2):213-28. Epub 2014/02/04.

2. Murray L, Henry A, Hoskin P, Siebert FA, Venselaar J. Second primary cancers after radiation for prostate cancer: a review of data from planning studies. Radiat Oncol. 2013;8:172. Epub 2013/07/10.

3. Schneider U, Zwahlen D, Ross D, Kaser-Hotz B. Estimation of radiationinduced cancer from three-dimensional dose distributions: Concept of organ equivalent dose. International journal of radiation oncology, biology, physics. 2005;61(5):1510-5. Epub 2005/04/09.

4. Murray LJ, Thompson CM, Lilley J, Cosgrove V, Franks K, Sebag-Montefiore $D$, et al. Radiation-induced second primary cancer risks from modern external beam radiotherapy for early prostate cancer: impact of stereotactic ablative radiotherapy (SABR), volumetric modulated arc therapy (VMAT) and flattening filter free (FFF) radiotherapy. Physics in medicine and biology. 2015;60(3):1237-57. Epub 2015/01/16.

5. Salembier C, Lavagnini $P$, Nickers $P$, Mangili $P$, Rijnders A, Polo A, et al. Tumour and target volumes in permanent prostate brachytherapy: a supplement to the ESTRO/EAU/EORTC recommendations on prostate brachytherapy. Radiotherapy and oncology : journal of the European Society for Therapeutic Radiology and Oncology. 2007;83(1):3-10.

6. Hoskin P, Rojas A, Ostler P, Hughes R, Alonzi R, Lowe G, et al. High-doserate brachytherapy alone given as two or one fraction to patients for locally advanced prostate cancer: acute toxicity. Radiotherapy and oncology : journal of the European Society for Therapeutic Radiology and Oncology. 2014;110(2):268-71.

7. Rivard MJ, Venselaar JL, Beaulieu L. The evolution of brachytherapy treatment planning. Medical physics. 2009;36(6):2136-53.

8. Schneider U, Sumila M, Robotka J. Site-specific dose-response relationships for cancer induction from the combined Japanese A-bomb and Hodgkin cohorts for doses relevant to radiotherapy (and associated erratum). Theoretical biology \& medical modelling [Internet]. 2011 [cited 2014 May 2]; 8:[27 p.]. Available from: http://www.tbiomed.com/content/pdf/1742-4682-8-27.pdf.

9. Schneider U. Mechanistic model of radiation-induced cancer after fractionated radiotherapy using the linear-quadratic formula. Medical physics. 2009;36(4):113843. Epub 2009/05/29.

10. Mazeron JJ, Scalliet P, Van Limbergen E, Lartigau E. Radiobiology of Brachytherapy and the Dose-Rate Effect. 2012 [21st May 2015]; Available from: http://www.estro.org/binaries/content/assets/estro/about/gec-estro/handbook-ofbrachytherapy/e-4-23072002-radiobiology-print proc.pdf

11. Grimm PD, Blasko JC, Sylvester JE, Meier RM, Cavanagh W. 10-year biochemical (prostate-specific antigen) control of prostate cancer with (125)I brachytherapy. International journal of radiation oncology, biology, physics. 2001;51(1):31-40. Epub 2001/08/23.

12. Zelefsky MJ, Hollister T, Raben A, Matthews S, Wallner KE. Five-year biochemical outcome and toxicity with transperineal CT-planned permanent I-125 
prostate implantation for patients with localized prostate cancer. International journal of radiation oncology, biology, physics. 2000;47(5):1261-6. Epub 2000/07/13.

13. Tselis N, Tunn UW, Chatzikonstantinou G, Milickovic N, Baltas D, Ratka M, et al. High dose rate brachytherapy as monotherapy for localised prostate cancer: a hypofractionated two-implant approach in 351 consecutive patients. Radiat Oncol [Internet]. 2013 [cited 2014 August 5]; 8:[115 p.]. Available from: http://www.rojournal.com/content/pdf/1748-717X-8-115.pdf.

14. Demanes DJ, Martinez AA, Ghilezan M, Hill DR, Schour L, Brandt D, et al. High-dose-rate monotherapy: safe and effective brachytherapy for patients with localized prostate cancer. International journal of radiation oncology, biology, physics. 2011;81(5):1286-92. Epub 2011/02/12.

15. Yoshioka Y, Konishi K, Sumida I, Takahashi Y, Isohashi F, Ogata T, et al. Monotherapeutic high-dose-rate brachytherapy for prostate cancer: five-year results of an extreme hypofractionation regimen with $54 \mathrm{~Gy}$ in nine fractions. International journal of radiation oncology, biology, physics. 2011;80(2):469-75. Epub 2010/07/22. 16. Nieder AM, Porter MP, Soloway MS. Radiation therapy for prostate cancer increases subsequent risk of bladder and rectal cancer: a population based cohort study. The Journal of urology. 2008;180(5):2005-9. Epub 2008/09/20.

17. Hinnen KA, Schaapveld M, Van Vulpen M, Battermann JJ, Van Der Poel H, Van Oort IM, et al. Prostate brachytherapy and second primary cancer risk: A competitive risk analysis. J Clin Oncol. 2011;29(34):4510-5.

18. Musunuru H, Mason M, Murray L, Al-Qaisieh B, Bownes P, Smith J, et al. Second primary cancers occurring after I-125 brachytherapy as monotherapy for early prostate cancer. Clin Oncol (R Coll Radiol). 2014;26(4):210-5. Epub 2014/02/11.

19. Zelefsky MJ, Housman DM, Pei X, Alicikus Z, Magsanoc JM, Dauer LT, et al. Incidence of secondary cancer development after high-dose intensity-modulated radiotherapy and image-guided brachytherapy for the treatment of localized prostate cancer. International journal of radiation oncology, biology, physics. 2012;83(3):9539. Epub 2011/12/17.

20. Liauw SL, Sylvester JE, Morris CG, Blasko JC, Grimm PD. Second malignancies after prostate brachytherapy: Incidence of bladder and colorectal cancers in patients with 15 years of potential follow-up. International journal of radiation oncology, biology, physics. 2006;66(3):669-73.

21. Moon K, Stukenborg GJ, Keim J, Theodorescu D. Cancer incidence after localized therapy for prostate cancer. Cancer. 2006;107(5):991-8.

22. Abdel-Wahab M, Reis IM, Hamilton K. Second primary cancer after radiotherapy for prostate cancer--a seer analysis of brachytherapy versus external beam radiotherapy. International journal of radiation oncology, biology, physics. 2008;72(1):58-68.

23. Huang J, Kestin LL, Ye H, Wallace M, Martinez AA, Vicini FA. Analysis of second malignancies after modern radiotherapy versus prostatectomy for localized prostate cancer. Radiotherapy and oncology : journal of the European Society for Therapeutic Radiology and Oncology. 2011;98(1):81-6.

24. Zelefsky MJ, Pei X, Teslova T, Kuk D, Magsanoc JM, Kollmeier M, et al. Secondary cancers after intensity-modulated radiotherapy, brachytherapy and radical prostatectomy for the treatment of prostate cancer: incidence and causespecific survival outcomes according to the initial treatment intervention. BJU international. 2012;110(11):1696-701. Epub 2012/08/15. 
25. Reddy C, Ciezki JP, Klein EA. Second malignancies after definitive radiotherapy for prostate cancer [abstract]. Brachytherapy. 2010;9:S78.

26. Takam R, Bezak E, Yeoh EE. Risk of second primary cancer following prostate cancer radiotherapy: DVH analysis using the competitive risk model. Physics in medicine and biology. 2009;54(3):611-25.

27. Dasu A, Toma-Dasu I, Olofsson J, Karlsson M. The use of risk estimation models for the induction of secondary cancers following radiotherapy. Acta Oncol. 2005;44(4):339-47. Epub 2005/08/27.

28. Schneider U. Modelling the risk of second malignancies after radiotherapy. Genes [Internet]. 2011 [cited 2013 June 11]; 2:[17 p.]. Available from: http://www.ncbi.nlm.nih.gov/pmc/articles/PMC3927608/pdf/genes-02-01033.pdf. 29. Fontenot JD, Bloch C, Followill D, Titt U, Newhauser WD. Estimate of the uncertainties in the relative risk of secondary malignant neoplasms following proton therapy and intensity-modulated photon therapy. Physics in medicine and biology. 2010;55(23):6987-98. Epub 2010/11/16.

30. Nguyen J, Moteabbed M, Paganetti H. Assessment of uncertainties in radiation-induced cancer risk predictions at clinically relevant doses. Medical physics. 2015;42(1):81-9.

31. Carrier JF, D'Amours M, Verhaegen F, Reniers B, Martin AG, Vigneault E, et al. Postimplant dosimetry using a Monte Carlo dose calculation engine: a new clinical standard. International journal of radiation oncology, biology, physics. 2007;68(4):1190-8.

32. Candela-Juan C, Perez-Calatayud J, Ballester F, Rivard MJ. Calculated organ doses using Monte Carlo simulations in a reference male phantom undergoing HDR brachytherapy applied to localized prostate carcinoma. Medical physics.

2013;40(3):033901 . 
Figure 1. Risks of second rectal or bladder cancer from LDR brachytherapy monotherapy relative to HDR brachytherapy monotherapy. Two different models have been used to estimate the risk of second rectal and bladder cancer for LDR brachytherapy.

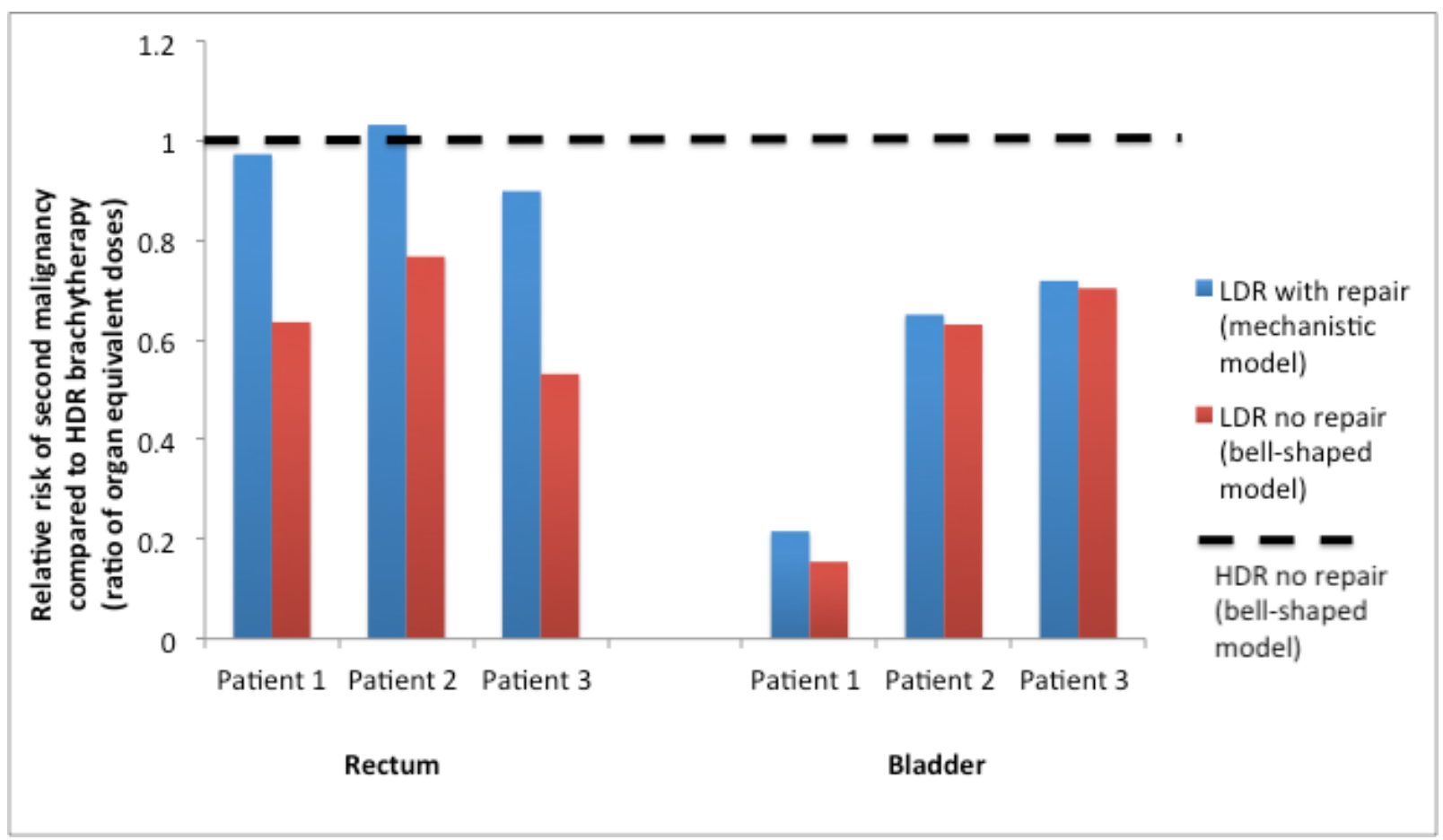


Figure 2. Excess absolute risk of second rectal or bladder cancer from LDR brachytherapy monotherapy and HDR brachytherapy monotherapy. Two different models have been used to estimate the risk of second rectal and bladder cancer for LDR brachytherapy.

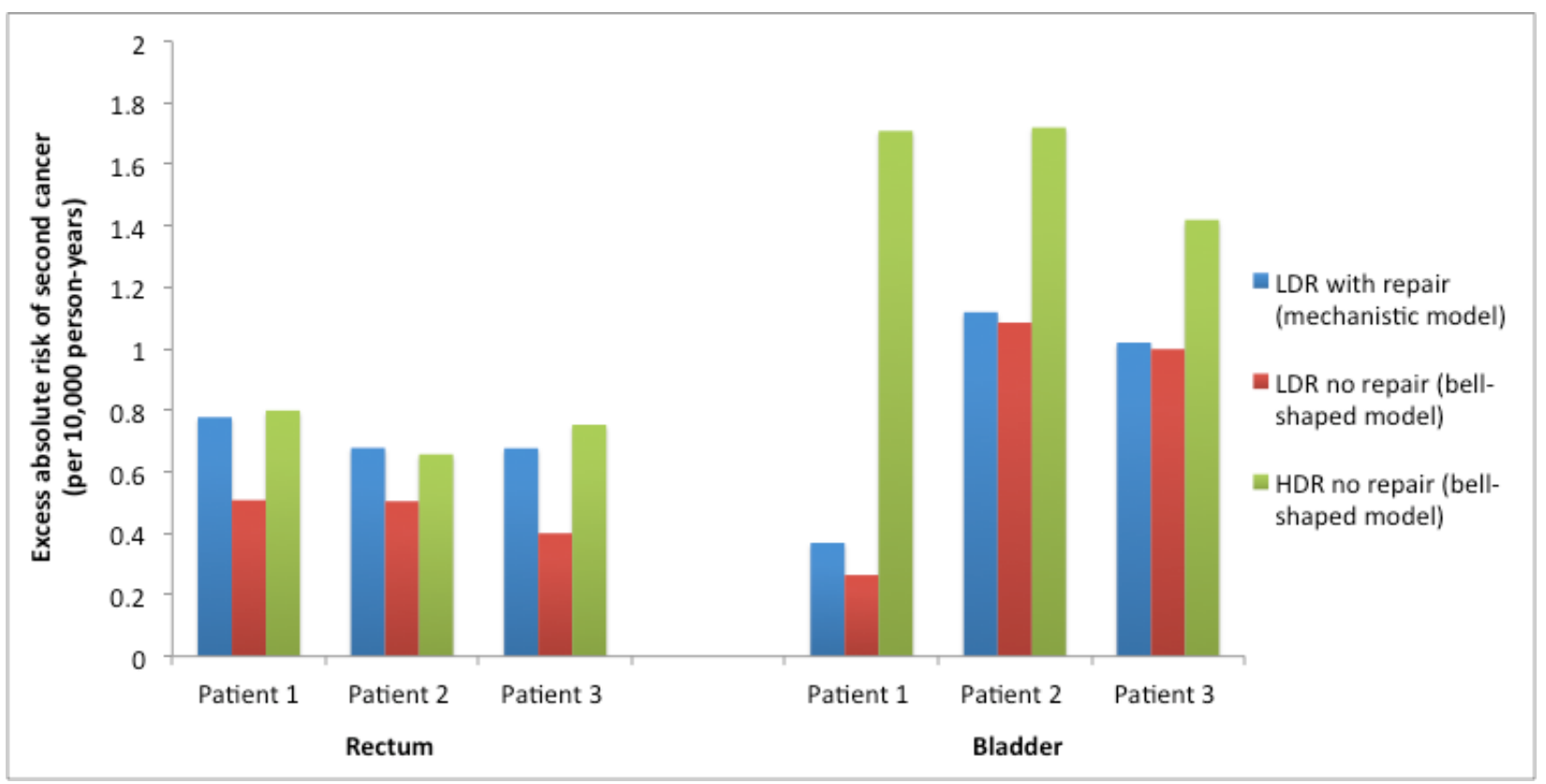


Figure 3. Risks of second rectal or bladder cancer from several radiotherapy techniques relative to 3D-confromal radiotherapy. Two different models have been used to estimate the risk of second rectal and bladder cancer for LDR brachytherapy.

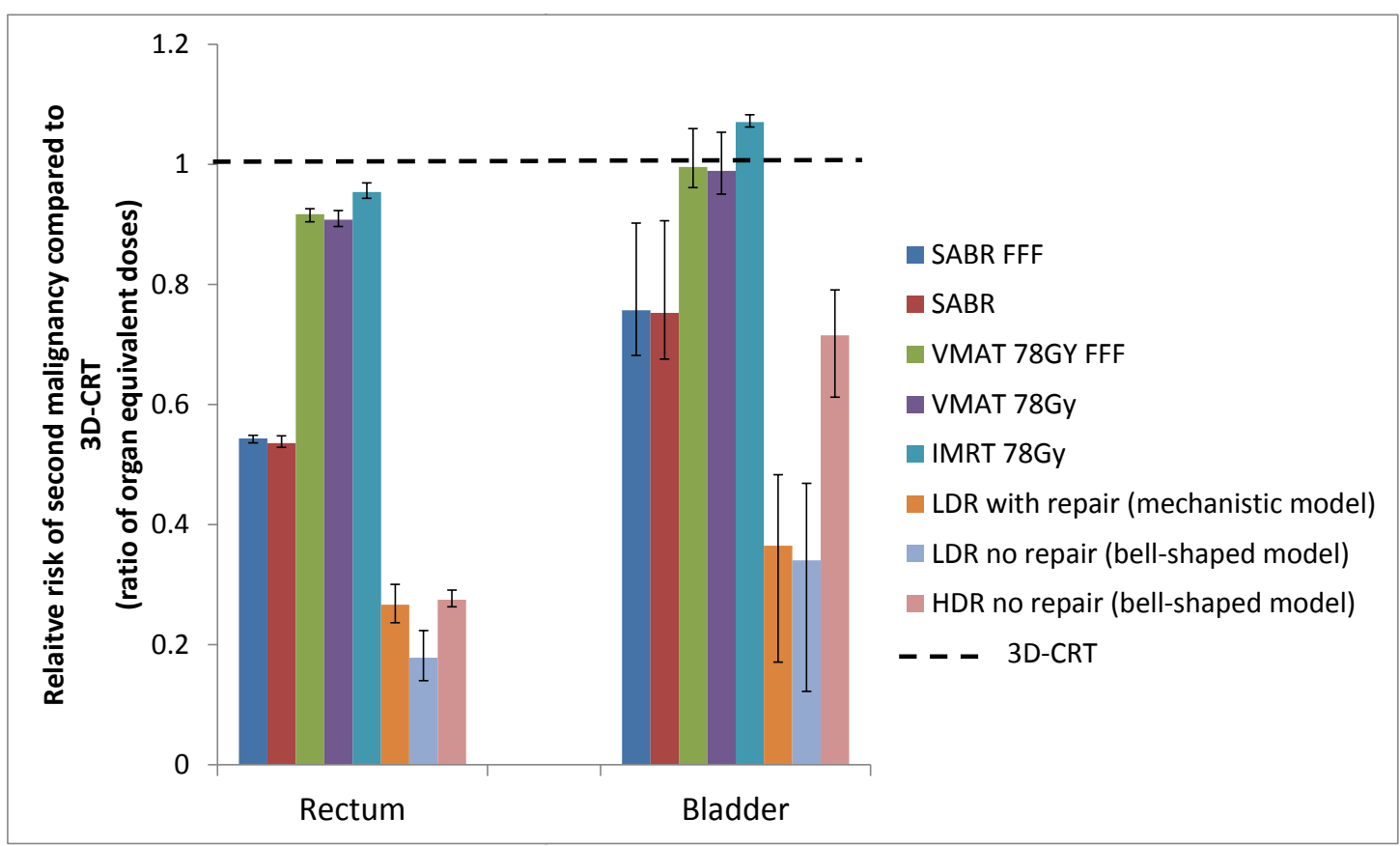

Average values for three plans displayed. Error bars display the range of values for all three evaluated plans. All external beam risks estimated using a mechanistic model. 
Figure 4. Excess absolute risk of second rectal or bladder cancer from several prostate radiotherapy techniques. Two different models have been used to estimate the risk of second rectal and bladder cancer for LDR brachytherapy.

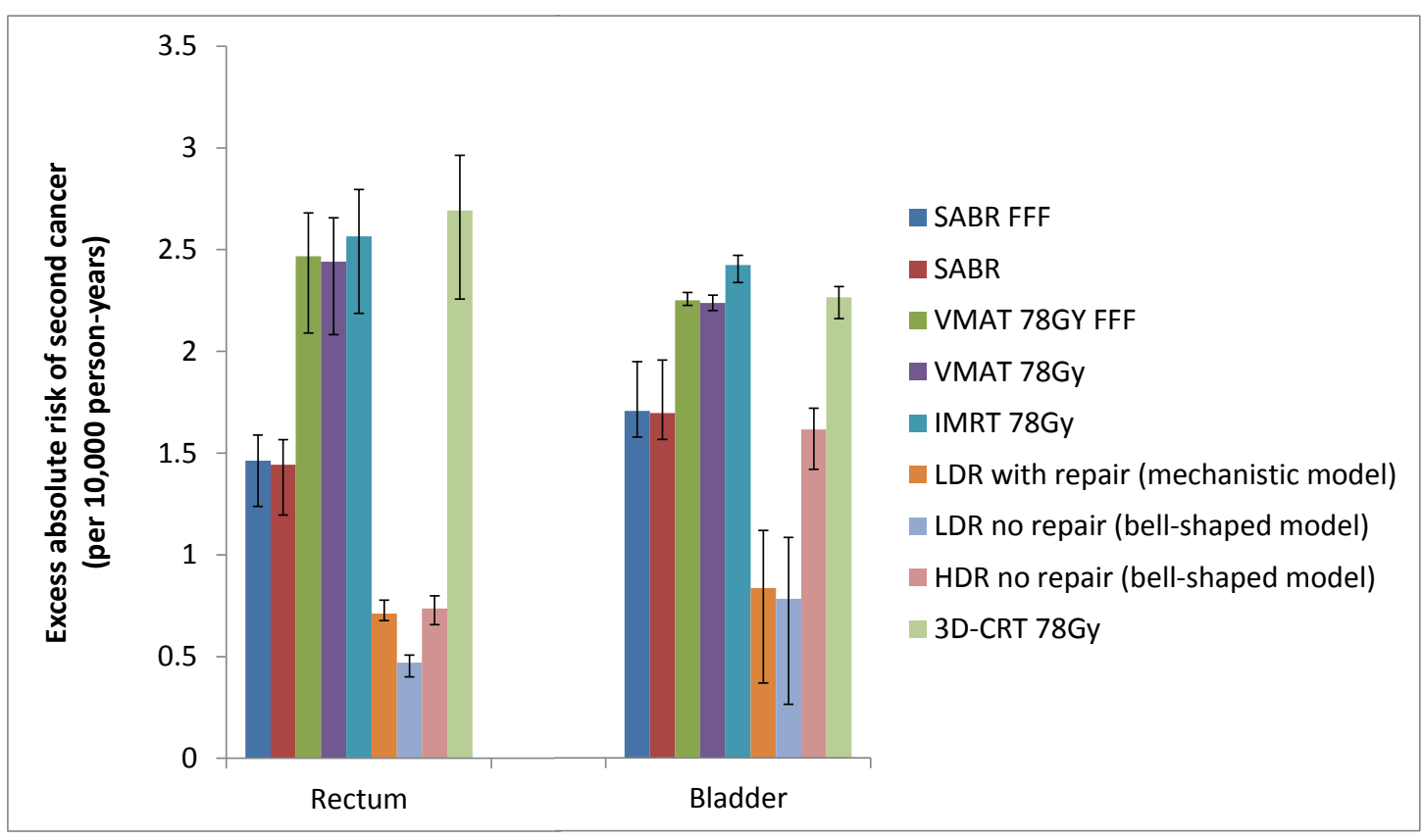

Average values for three plans displayed. Error bars display the range of values for all three evaluated plans. All external beam risks estimated using a mechanistic model. 


\section{Supplementary Material}

Figure 1. Does-response relationship for rectum (Figure 1a) and bladder (Figure 1b) for single fraction brachytherapy according to Schneider's mechanistic model (repair permitted) and bell-shaped model (no repair) [1]. The vertical axis is intentionally left blank but reflects the Risk Equivalent Dose as described in the main manuscript.

Figure 1a) Rectum

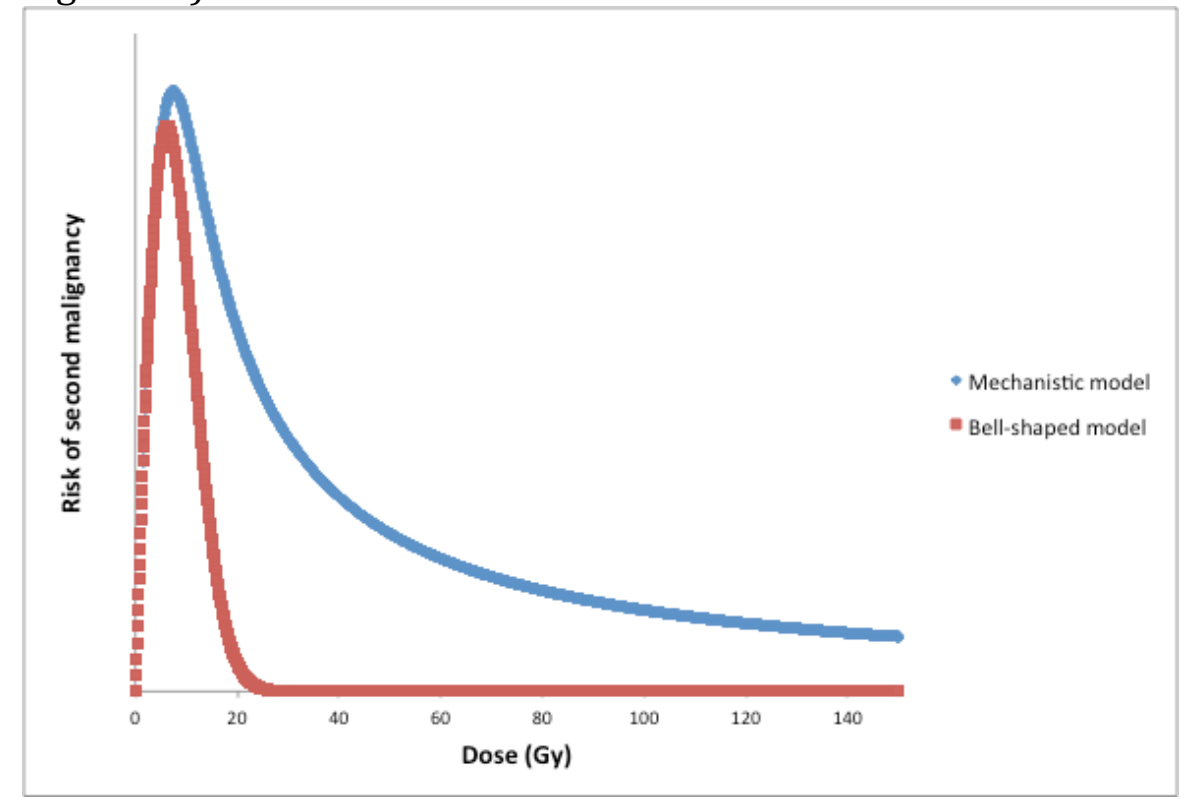

Figure 1b) Bladder

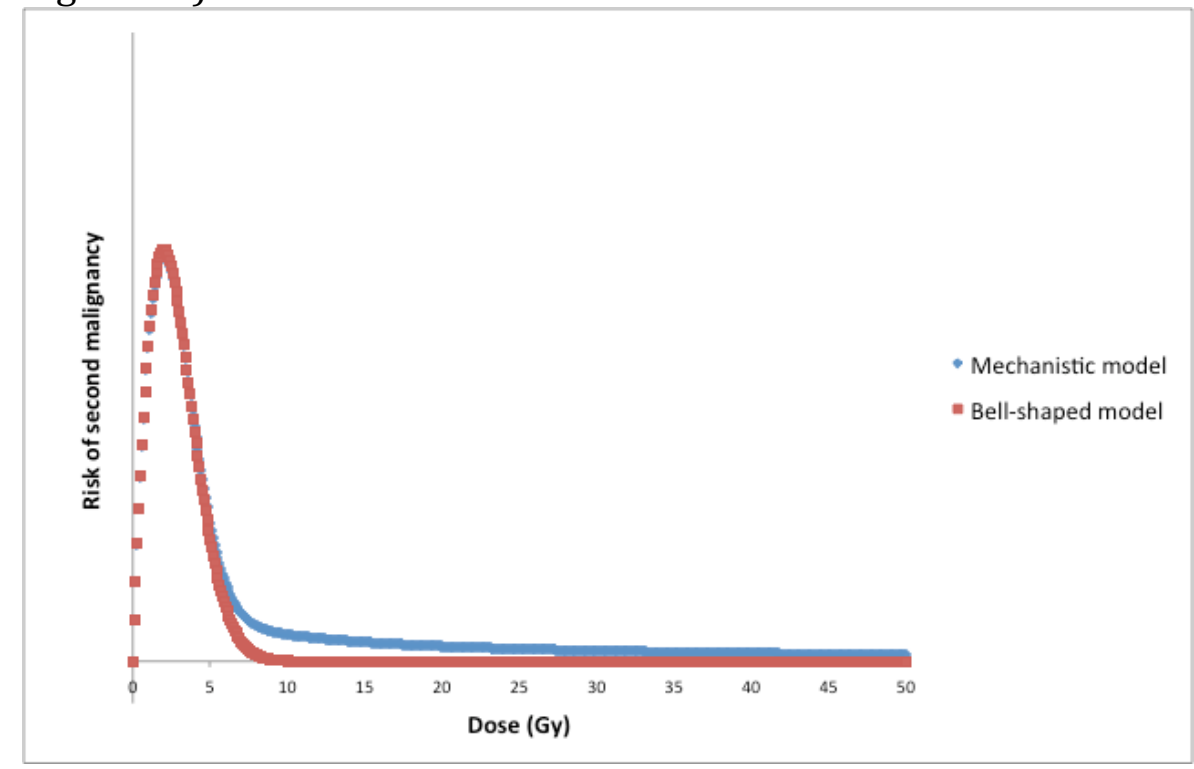


1. Schneider U, Sumila M, Robotka J. Site-specific dose-response relationships for cancer induction from the combined Japanese A-bomb and Hodgkin cohorts for doses relevant to radiotherapy (and associated erratum). Theoretical biology \& medical modelling [Internet]. 2011 [cited 2014 May 2]; 8:[27 p.]. Available from:

http://www.tbiomed.com/content/pdf/1742-4682-8-27.pdf. 